\title{
Augmented Reality in Enhancing Qualitative Education
}

\author{
Suvarna Kumar Gogula, \\ PhD \\ Associate Professor \\ MVGR college of Engineering \\ Vizianagaram
}

\author{
Sandhya Devi Gogula, \\ $\mathrm{PhD}$ \\ Assistant Professor \\ MVGR college of Engineering \\ Vizianagaram
}

\author{
Chanakya Puranam \\ MVGR college of Engineering \\ CSE Department \\ Vizianagaram
}

\begin{abstract}
Augmented Reality plays a prominent role in transforming education into innovative ways as it has the capacity of overlaying rich virtual world which can coordinate with real world. Augmented Reality also provides mobility for its applications which makes students to easily adopt this kind of innovative learning. It provides students with 'Perfectly Situated Scaffolding' [1]. This paper presents the significance of Augmented Reality in the field of education through mobile devices which allow the students to have a new way of interactive classroom teaching. In the classroom, camera scans the markers and projects the objects into virtual world by mapping the $3 \mathrm{D}$ coordinates. The presented application helps students to improve the way of learning by visualizing the 3D world and makes the students to interact with the virtual objects. This paper also discusses about the present day classroom learning and learning with augmented reality and the way of implementing it. A couple of feedbacks were taken from students after presenting a augmented reality class in which different topics were explained with various scenarios of virtual objects. The report from students demonstrates the significant change in student's perception towards effective learning of concepts. Finally this paper concludes by giving a large scope for research in improving the quality of present day education system by employing augmented reality concept.
\end{abstract}

\section{General Terms}

Fragmentation, Coordinates ,Class Rooms

\section{Keywords}

Augmented Reality, Markers, Virtual Objects

\section{INTRODUCTION}

Augmented Reality allows user to track the virtual world with the user. The main objective of augmented reality is to improve and enhances perfection of surroundings by combining, sensing .So Augmented reality applications can combine virtual and real world, Interactive in real time, Registered for 3D[2].Augmented reality besides its features it is applicable to different fields like Architecture, Gaming, Education, Navigation, Tourism and many more[3].Augmented Reality can be applied in different ways like marker based, voice based, skeletal based, gesture based sby using different tools like Vuforia, Wikitude, Kinect, OpenSpace3D and many more tools. The present application uses an open source tool - OpenSpace3D.

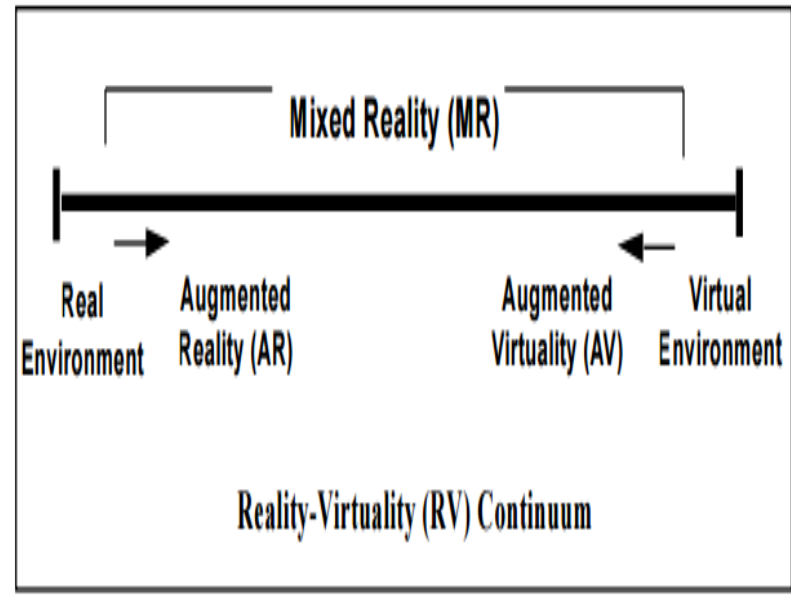

Figure 1: Milgram's Reality-Virtuality Continuum [4]

\subsection{Augmented Reality in OpenSpace3D}

Augmented Reality can be done by using different free as well as commercial tools, OpenSpace 3D is one of such tools which is developed by I-maginer.OpenSpace3D provides different options like Face Tracking, Marker Tracker, Skeletal Tracking which are the main part of Augmented Reality and OpenSpace3D provides a lot of 3D meshes which were the part of Virtual World and supports different smart devices and sensors like Kinect, MYO, Leap Motion and many more.

In OpenSpace3D, Users can interact with the virtual environment in various ways, which are:

- Navigate in the virtual environment

- Visualize the training courses from the gallery

- Visualize DCNS medias

- Display and manipulate DCNS products (models)

- Visualize the planning

- Visualize monitoring content

- Consult messages

- Chat with other students and with professors

- Switch to LMS web application[5] 


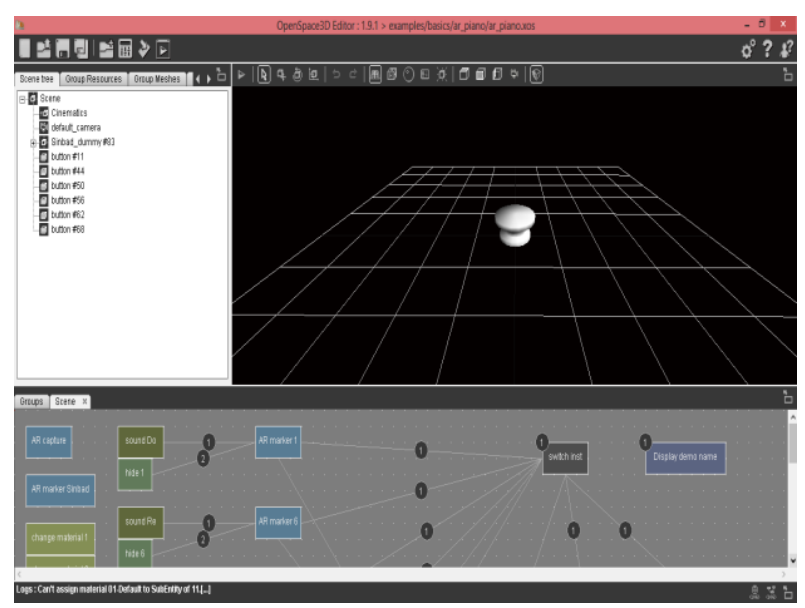

Figure 2: OpenSpace3D

\section{WORK IN AUGMENTED REALITY}

\subsection{Problem Definition}

As most of the students face the problem of understanding when there is a 3D figure explanation but the book gives a $2 \mathrm{D}$ model which a teacher uses it to explain and more over whatever the students/learners read mostly will be imaginary and student need to make picture view in his mind. In such cases, most of the students fail in such kind of thinking which makes them to lose their practical visualization. Sometimes it takes out the student interest on the subject or changes the student's perception towards learning a subject.

So from the subject/knowledge point of view, Augmented Reality provides some kind of imaginary world to the student respective of the topic he is going through which makes students to analyze and specify the subject and gains the knowledge

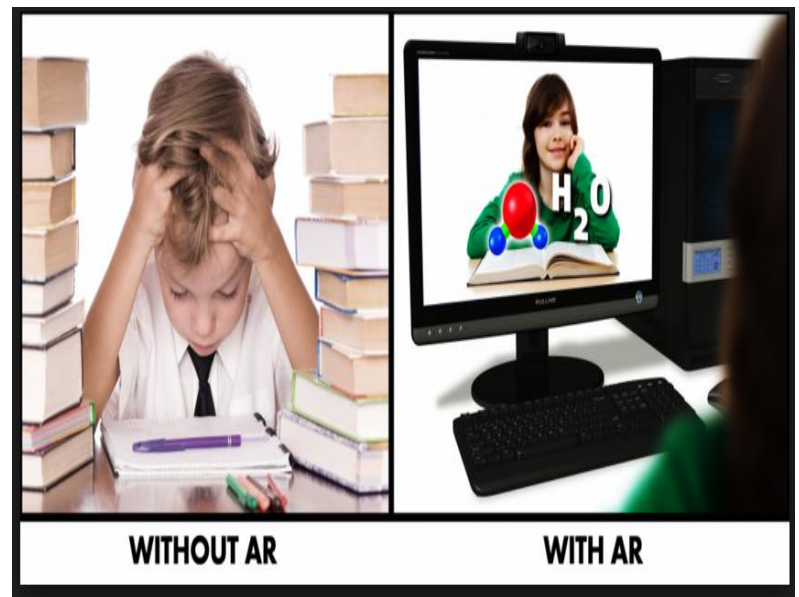

Figure 3: Education with and without AR [6]

\subsection{Previous Work}

In the classrooms till date, teaching has been moved from blackboard to projector where teacher presents lot of presentations and videos which also helps the students to get practical exposure. But there may be some muddles in student's minds of how the topic is related to him?, what are the real time scenarios in which the related topics are applicable?. These are what present day class room teaching is missing. Moreover, these all kinds of teaching don't provide a practical exposure and more over in some specializations for making a practical work it costs more and very difficult to bear the cost.

\subsection{Present Work}

As from previous discussion, students are lacking how a concept can be applied in the real scenario for example: fragmentation in networks students know what it, what are the formulas related to it is. So this application focuses on that example only on how the fragmentation applied in the class room how it works when student himself/herself sends a message, what is the process will be happened those are imaginary ones/virtual world will be coming in the real world of a class room using the Augmented Reality. Through these applications of Augmented Reality helps the students to visualize the tiny as well as nano objects easily. These kind of AR applications helps not only for professional students but also the small children in their way of learning. These AR applications are very much mobilizes so they can be work in any platform

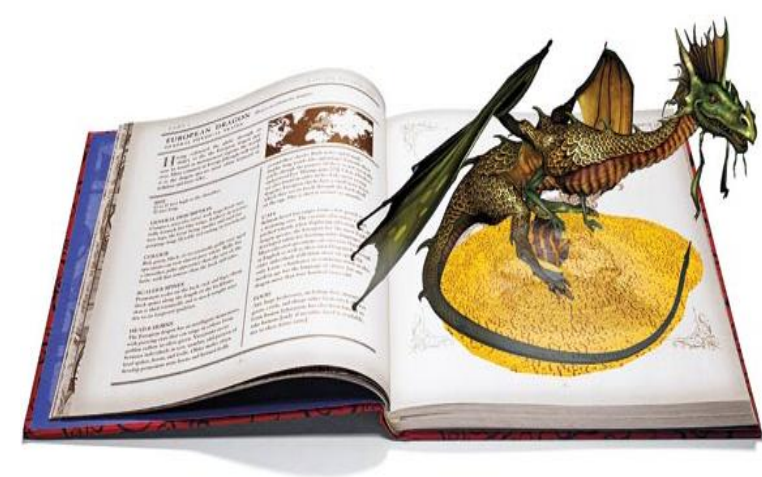

Figure 4: The way of projecting virtual world [7]

Here in these kind of applications only virtual world changes but the way of developing is same. It is like class which is fixed and encapsulated, only change is the two objects one is marker and its corresponding virtual object.

\section{DIFFERENT WAYS OF AUGMENTED REALITY IN EDUCATION}

Augmented Reality in the field of Education can be implemented in many ways like Marker Based, Geo Location Based, Skeletal based, Gesture based. But whatever is the way of implementation the method is too similar. All the above mentioned ways differ in the inputs like markers, gestures, voice but after getting the input and detecting the input of the user obviously projects a virtual world which interacts with the user.

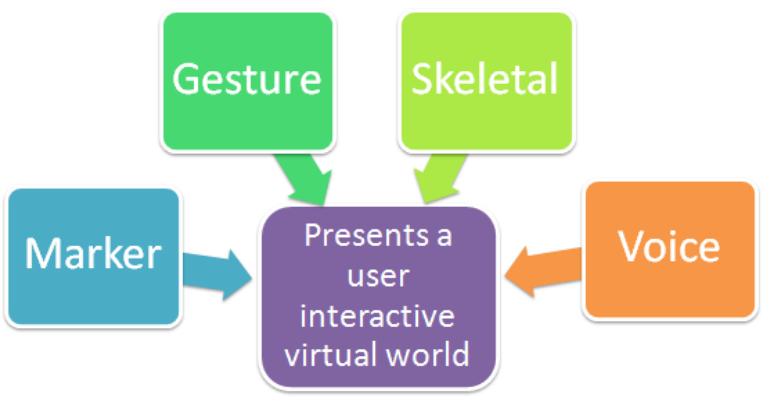

Figure 5: Model of Augmented Reality 


\subsection{Voice based Augmented Reality}

In this way of augmented reality, here depending on the voice based input from the user the virtual world changes. Here there will be a set of words which are associated with the virtual world. Here there is a problem with the pronunciation, but not for all cases. In education through AR, voice based Augmented reality will give good results but not all the times

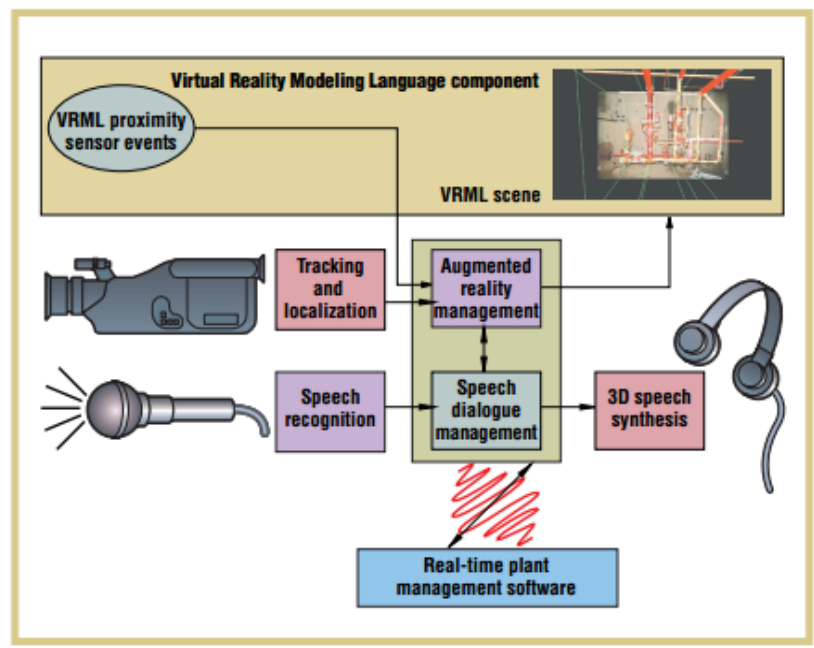

Figure 6: Voice based Augmented Reality [8]

\subsection{Skeletal based Augmented Reality}

In this way of augmented reality, here depending on the different skeletal actions tracking will be done and for every particular skeletal action there lies a virtual world which will be projected. Here in Education, Skeletal tracking will give good results but expensive

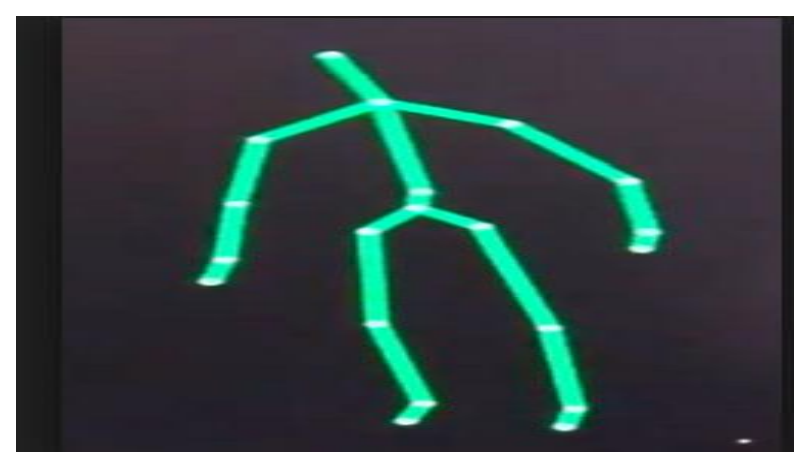

Figure 7: Skeletal Based Augmented Reality [9]

\subsection{Gesture Based Augmented Reality}

In this way of augmented reality, here depending on the different gestures tracking will be done and for every particular gesture there lies a virtual world which will be projected. Here in Education, gesture tracking will give best results both in the classroom as well as student while studying but its complex and expensive

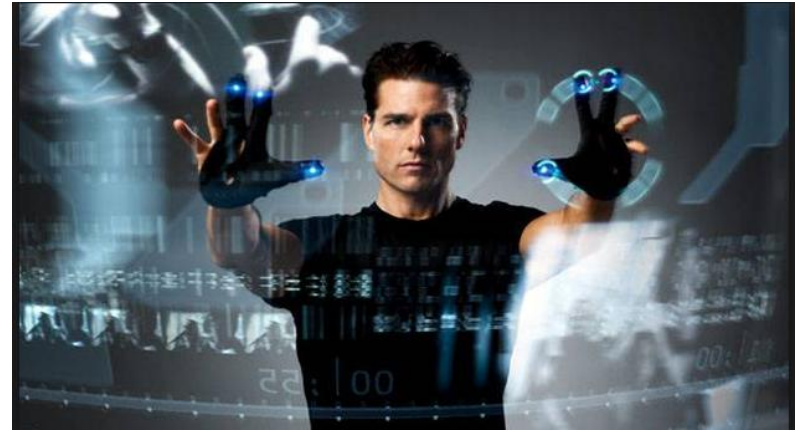

Figure 8: Gesture Based Augmented Reality [10]

\subsection{Marker based Augmented Reality}

Marker based AR has been discovered a decade ago. There are different tools available for marker based AR where the model of implementation is same and simple. There are some markers in the dataset and each marker associated with some virtual world interaction, once the application starts camera scans for the markers once the marker is detected with the help of some image recognition techniques, virtual world will be projected and user can interact with it.

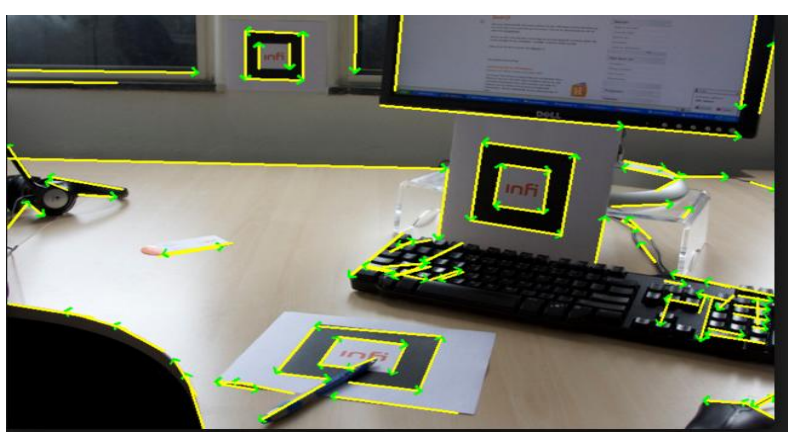

Figure 9: Marker Based AR [11]

\section{Setting a Augmented Reality classroom}

To demostrate the idea, an augmented reality application is created which uses the camera tracks the markers, users and their faces, augments the virtual world and makes them to interact with real world objects by mapping all the 3D Coordinates. Also, marker based augmented reality with some of the keyboard shortcuts were used to augment the virtual world.

\subsection{Markers in Application}

In this marker-based AR application the images (or the corresponding image descriptors) are provided beforehand. In this case you know exactly what the application should recognize while acquiring camera data. Most of the nowadays AR apps dealing with image recognition are marker-based. [12]. Here in this application, different makers used are pictures, names to track the real world and mapping with virtual world.

Markers play a major role in tracking the real world so more care to given and it uses image processing algorithms to recognize the image. 


\section{FRAGMENTATION}

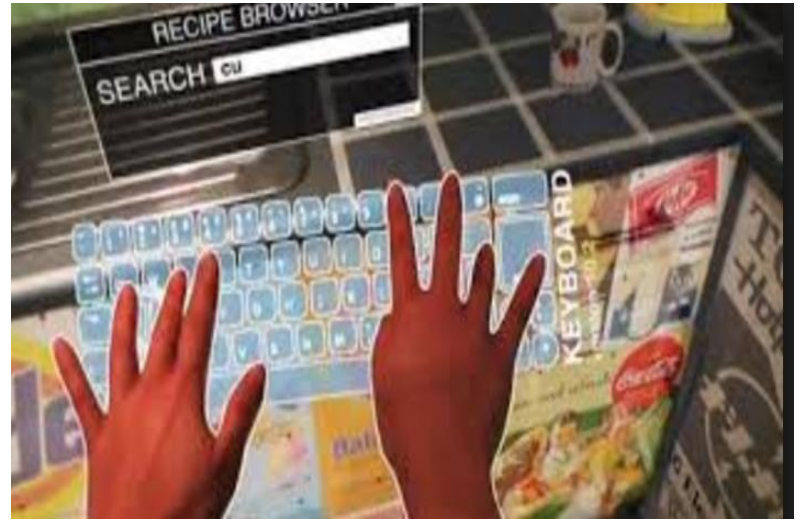

Figure 12: Mapping Virtual and Real world

\subsection{Lighting Conditions}

To create a more realistic environment, the lighting conditions of the RGB image needs to be taken care of. This is done by converting the image to an HSV image and taking an average of the intensity from the user [13].

\section{IMPLEMENTATION}

In this application, a tool called OpenSpace3D is used which is a free and Open Source development platform for interactive real time 3D projects [14].

Here on starting the application, camera will be called by using AR Capture in OpenSpace3D, then camera scans for markers and students with the help AR Marker and AR Face Tracker and once markers are detected virtual world will be projected which will be connected to a normal or hologram projector which projects the complete scene and makes student to analyze practically. Through this OpenSpace3D, this application can be exported as an Android Application, Web Application or Standalone Windows Application.

There are many other tools to design such applications but as OpenSpace3D is open source and even more plugIn's are available through which different marker based applications can be made effectively.

\section{RESULTS}

The application designed is exhibited in a class room comprising of 60 students. Feedback was taken from them based on two particulars. One is about the classroom learning with and without AR and other is about how different virtual objects make effective learning possible.

\subsection{Classroom Learning with and without \\ AR}

Table 1 presented below represents the data from students after experiencing AR classroom teaching. Students gave grades for 1 to 5 to both classroom experiences with and without AR.

Table 1: Student grades after listening to a concept with $A R$ and without $A R$. in marker virtual objects will be affected. When the marker is found the 3D coordinates, mapping plays a major role when it comes to interaction with real world. Their way of rotation, scaling and their position everything matters as if something went wrong in mapping, the entire essence of augmented reality will be lost. So initially, projection of virtual world needs to be done. For video objects, a flat projection over the students is made where as with 3D models on interaction location changes mainly because orientation of 3D objects change rapidly.

\begin{tabular}{|c|c|c|}
\multicolumn{4}{|c|}{ AR and without AR. } \\
\hline Grades & With AR & Without AR \\
\hline 5 & 17 & 1 \\
\hline 4 & 24 & 5 \\
\hline 3 & 15 & 18 \\
\hline 2 & 3 & 21 \\
\hline 1 & 1 & 15 \\
\hline
\end{tabular}




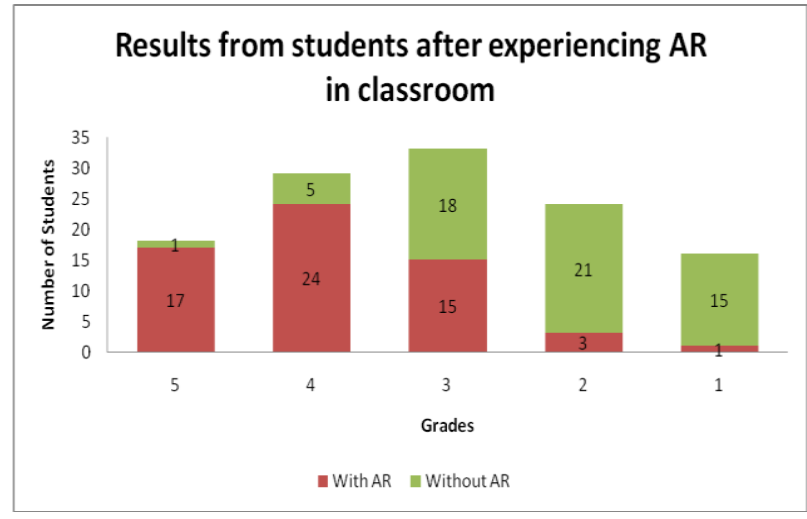

Fig 13: Graphical representation of table 1.

\subsection{Effect of Virtual Objects In AR Classroom}

Table 2 represents the feedback from students rating how different virtual objects like video, 3D objects and photos brought the effectiveness of AR. Similar to previous results students rated from 1 to 5 for each of the three virtual objects.

Table 2: Student grades for effectiveness of various virtual objects in classroom environment.

\begin{tabular}{|c|c|c|c|}
\hline Grades & $\begin{array}{c}3 \text { D } \\
\text { Objects }\end{array}$ & Video & Photo \\
\hline 5 & 29 & 24 & 7 \\
\hline 4 & 19 & 15 & 8 \\
\hline 3 & 9 & 10 & 21 \\
\hline 2 & 2 & 7 & 9 \\
\hline 1 & 1 & 4 & 15 \\
\hline
\end{tabular}

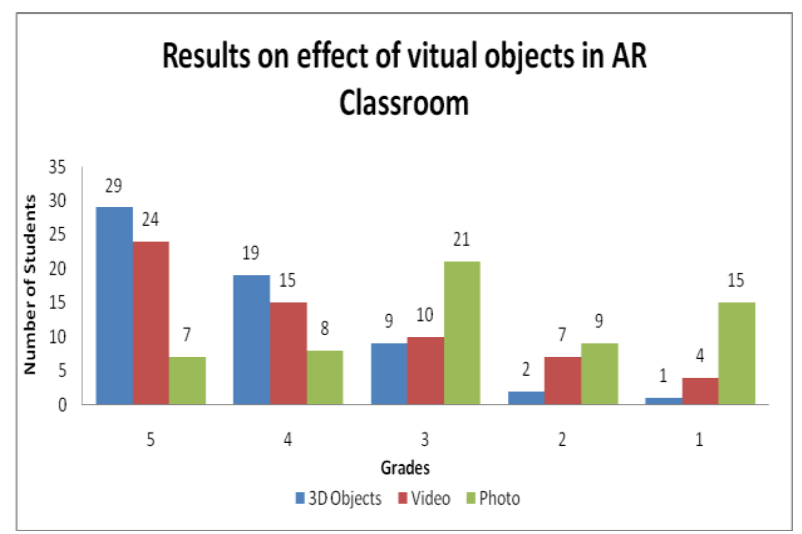

Fig 14: Graphical representation of Table 2.

From the graphs, it is shown that student perspective of learning a concept through augmented reality is more effective compared to learning with conventional methods. Thus, it is demonstrated that augmented reality is an effective way in enhancing qualitative education.

\section{FUTURE SCOPE}

Through the studies made on Augmented Reality, it can be realized that there is a great scope for augmented reality applications in different areas like Archeology, Arts, Engineering, Education, Game Development, Product branding and many more[13]. Every representative way of augmented reality from voice to gesture has its own privilege in further development. Even one with good knowledge in multimedia can start a business on augmented reality and can work on education, product advertisements, Filming and many more.

\section{REFERENCES}

[1] Matt Bower, Cathie Howe, Nerida McCredie, Austin Robinson, David Grover AUGMENTED REALITY IN EDUCATION - CASES, PLACES, AND POTENTIALS

[2] http://www.csc.kth.se/ alx/courses/DT2140/olwal_introd uction_to_ar_2010_03_05.pdf

[3] https://en.wikipedia.org/wiki/Augmented_realityTavel, P. 2007 Modeling and Simulation Design. AK Peters Ltd.

[4] http://web.cs.wpi.edu/ gogo/hive/papers/Milgram_Take mura_SPIE_1994.pdf

[5] http://www.openspace3d.com/lang/en/newsactualites/

[6] http://www.twoguysandsomeipads.com/2013_08_01_arc hive.html

[7] http://larisamoniz.pbworks.com/w/page/36890577/Prese nt $\% 20$ Uses $\% 20$ for $\% 20$ Augmented $\% 20$ Reality $\% 20 \mathrm{in} \% 2$ 0Education

[8] https://www.cs.cmu.edu/ 15821/CDROM/PAPERS/goose2003.pdf

[9] http://ogata-lab.jp/ja/tech_ja/1994.html

[10] http://www.illawarramercury.com.au/story/1217747/ augmented-reality-in-a-hands-free-2013/

[11] https://infi.nl/nieuws/marker-detection-for-augmentedreality-applications/

[12] Shreya Kamani, Neel Vasa, Kriti Srivastava, D. J. "VIRTUAL TRIAL ROOM USING AUGMENTED REALITY" Sanghvi College of Engineering, Mumbai 53

[13] https://en.wikipedia.org/wiki/Augmented_reality

[14] Ausra Kalantiate, Aimuntas Kazimieras Parseliunas, Denis Romanovas, Dominykas Slikas, "Generating the open space D model based on LiDAR data" pages 152156 